\title{
Pullout Capacity Of Cylindrical Block Embedded In Sand
}

https://doi.org/10.2478/sgem-2018-0005

received October 16, 2017; accepted December 13, 2017.

Abstract: Calculation of pullout capacity of anchoring concrete cylindrical block by finite element method is carried out. 3D model of the block assumes its free rotation. Alternative solutions with one and two pulling forces attached at different heights of the block are considered. Dependency of the ultimate pulling force on the points of its application, the block's embedment depth as well as contact friction are investigated. Results of FE analysis and simple engineering estimations are compared. The maximum pullout resistance results from FE analysis when the rotation of the block is prevented.

Keywords: block anchors; pullout capacity; FE analysis; plastic limit analysis.

\section{Introduction}

Retaining walls of various constructions are often anchored. Anchored walls are usually more economical and of greater heights, unobtainable without reinforcement with anchors, than classical cantilever walls [1-4]. The anchoring element may be, as often used by engineers, a cylindrical concrete block situated at a certain distance from the wall. The cylindrical concrete block may be constructed from precast concrete tubes filled with concrete.

This paper provides finite element analysis [5] of the ultimate pullout capacity acting at different heights of the cylindrical block. The point of force application significantly influences the geometry of the failure zone

\footnotetext{
*Corresponding author: Krzysztof Sternik, Silesian University of Technology, Faculty of Civil Engineering, ul. Akademicka 5, 44-100 Gliwice, Poland, E-mail: krzysztof.sternik@polsl.pl Katarzyna Dołżyk-Szypcio: Bialystok University of Technology, Faculty of Civil and Environmental Engineering, ul. Wiejska 45E, 15351 Bialystok, Poland
}

in soil surrounding the block and the ultimate pullout force value. The ultimate pullout capacity of the plate anchors, essential in engineering, has been tested both experimentally [6,7] and theoretically [8-13]. The tests, however, concerned the ultimate pullout capacity of the plate anchors moving without rotation in the direction of the acting force. The paper presents results of the computational ultimate pullout capacity analyses of the cylindrical block rotating during displacement. The relationships between the anchoring block's dimensions, depth of embedment, points of pullout force application and pullout capacity have been shown. It has been proved that the location of the force applied to the cylinder has influence on its resistance.

\section{Problem description}

Anchors provide additional support to the upper part of an embedded wall. In that kind of wall, its base is embedded in the natural ground below the excavation bottom or the original ground level. In the most traditional sense, the anchor can be made up of an anchor block attached to a retaining wall with a tendon. After the anchors have been constructed, the ground is re-profiled with backfill. A pre-formed wall where there is no excavation during wall construction is shown in Fig. 1. Such a solution was proposed for the extension of the terrain surrounding the newly constructed warehouse. Fig. 2 presents the natural slope in the vicinity of the warehouse under construction and the completed lower part of the retaining wall. The natural ground in the slope is made of non-cohesive soils like fine and medium sand with local addition of coarse gravel in medium dense and dense state. Determination of pullout resistance was an integral and crucial part of the design.

A cylindrical block of diameter $D$ and height $H$ is embedded in sand at a depth $z$, below the horizontal soil level with no additional load (Fig. 3). It is assumed that the block is dragged horizontally by one horizontal, entirely 


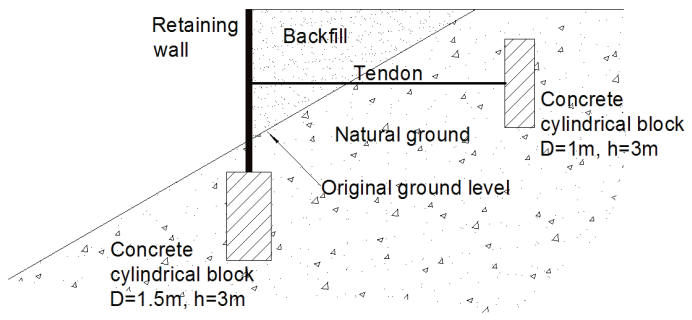

Figure 1: Anchored embedded retaining wall.

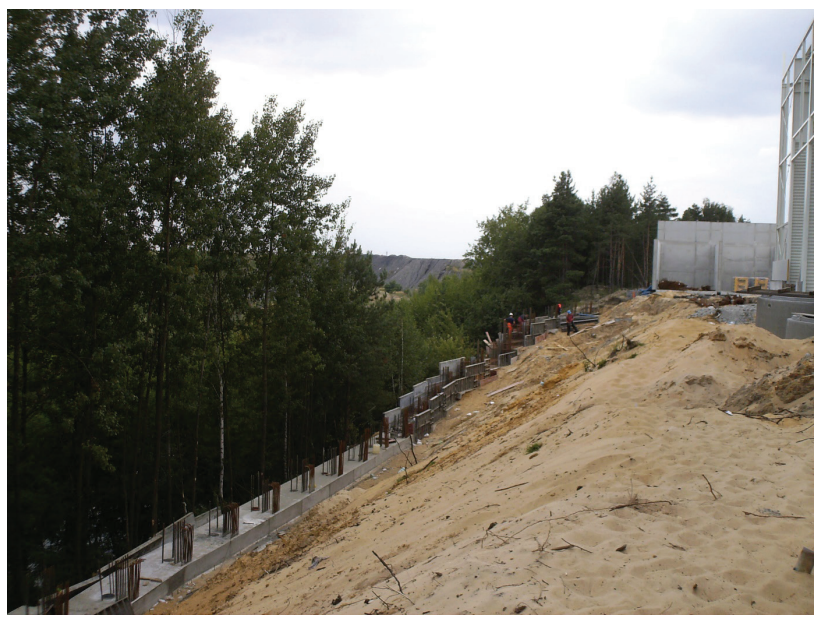

Figure 2: Embedded wall under construction.

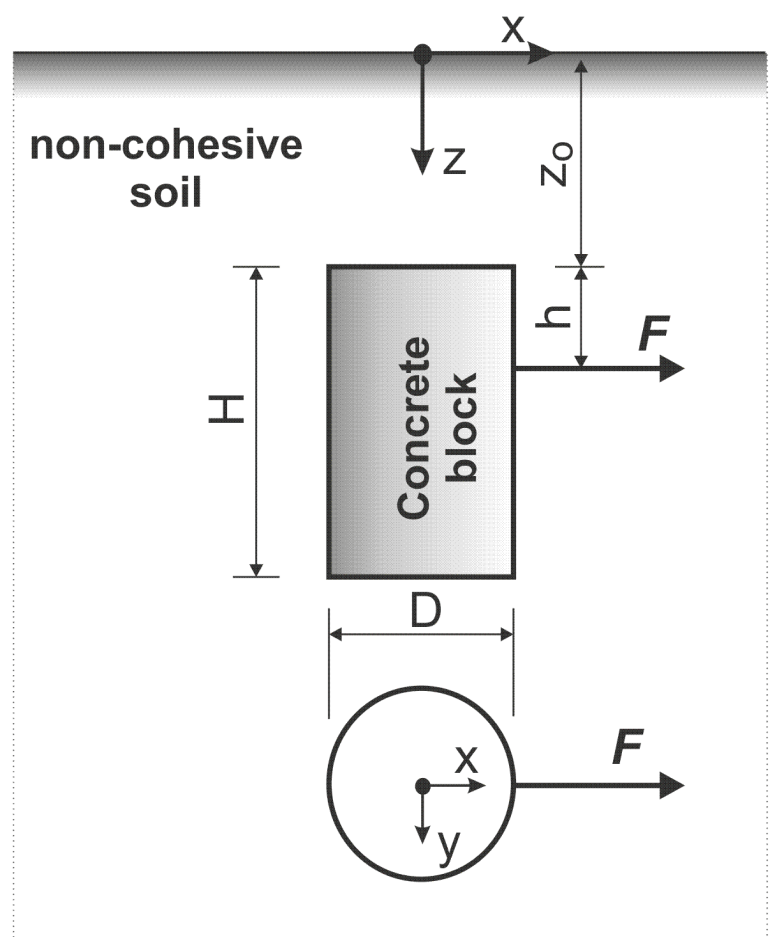

Figure 3: Geometry of the problem
Table 1: Material parameters.

\begin{tabular}{llll}
\hline & & sand & concrete \\
\hline Young Modulus & $E[\mathrm{MPa}]$ & 80 & 30000 \\
Poisson's ratio & $v[-]$ & 0.25 & 0.20 \\
Cohesion & $c[\mathrm{kPa}]$ & 0.0 & - \\
Friction angle & $\Phi\left[^{\circ}\right]$ & 34 & - \\
Unit weight & $\gamma\left[\mathrm{kN} / \mathrm{m}^{3}\right]$ & 18 & 25 \\
\hline
\end{tabular}

flexible tendon. The tendon is attached at a distance $\mathrm{h}$, below the block's top surface.

The horizontal ground surface is not loaded. The elastic - perfectly plastic Mohr-Coulomb model was assumed for the sand surrounding the block and the linear elastic model for concrete of the anchor block. The parameters of both models are listed in Table 1.

Volume changes of soil at yielding are reflected by the value of dilatancy angle $\psi$. Different behaviours of soil during shearing were taken into account in calculations by assuming incompressible yielding with $\psi=0^{\circ}$, as well as the increase in volume with $\psi=5^{\circ}$ and $10^{\circ}$. The influence of dilatancy angle on ultimate pullout capacity was tested.

Initial stress state before the application of the pullout force is given by $\sigma_{z}=\gamma z, \sigma_{x}=\sigma_{y}=K_{o} \sigma_{z}$, where $K_{o}=1-\sin \Phi$. Thus, the soil is assumed to be in a dry state.

Two thicknesses of the soil layer over the top of the block $\mathrm{z}_{0}=1 \mathrm{~m}$ and $2 \mathrm{~m}$ were considered in calculations. In FE models the following contact conditions between the block and the soil were taken into account: full contact with no interface elements on the block's surface as well as sliding and separation due to interface elements [14,15] between the block and the soil, with friction angle related to the internal friction angle of sand $\Phi=34^{\circ}: \delta=0,1 / 3 \Phi$ $\left(11.3^{\circ}\right), 2 / 3 \Phi\left(22.7^{\circ}\right)$, $\Phi$.

The cylindrical block is treated as a very strong element; thus, it is assumed to be described by linear elasticity.

\section{Finite element model and boundary conditions}

The three-dimensional model of the soil mass in which the concrete block is embedded is shown in Fig. 4. The discretization of the domain considered was done with 

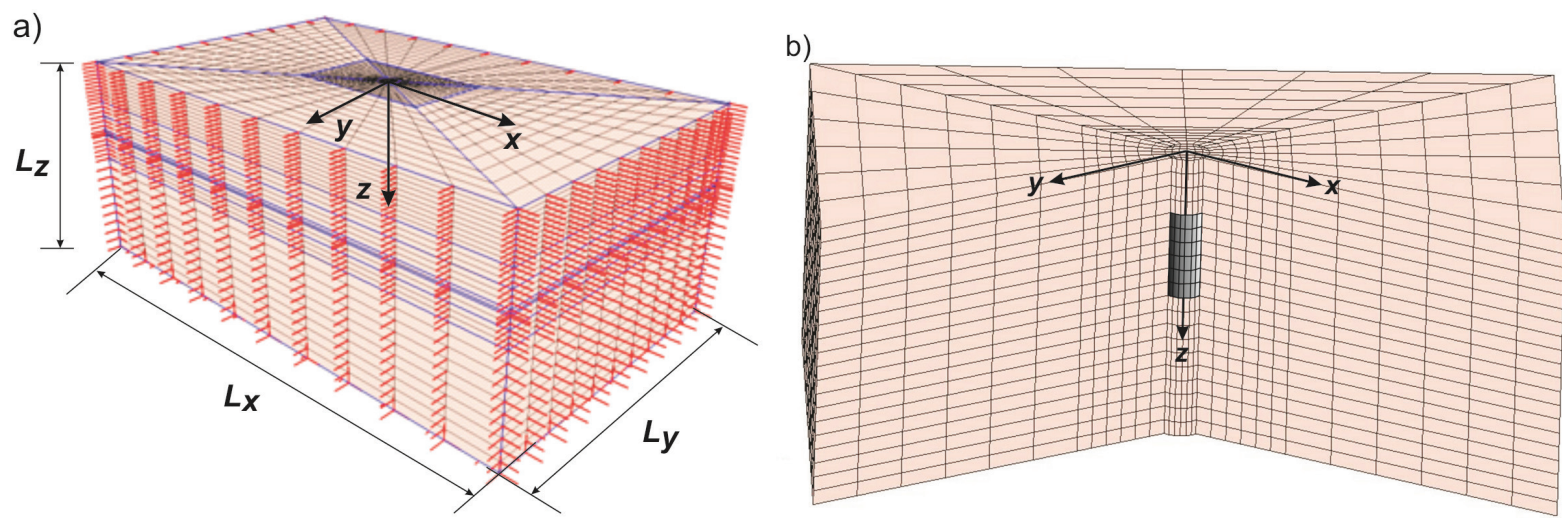

Figure 4: Finite element mesh: a) general view; b) cross-section of the model for $z_{0}=2 \mathrm{~m}$.

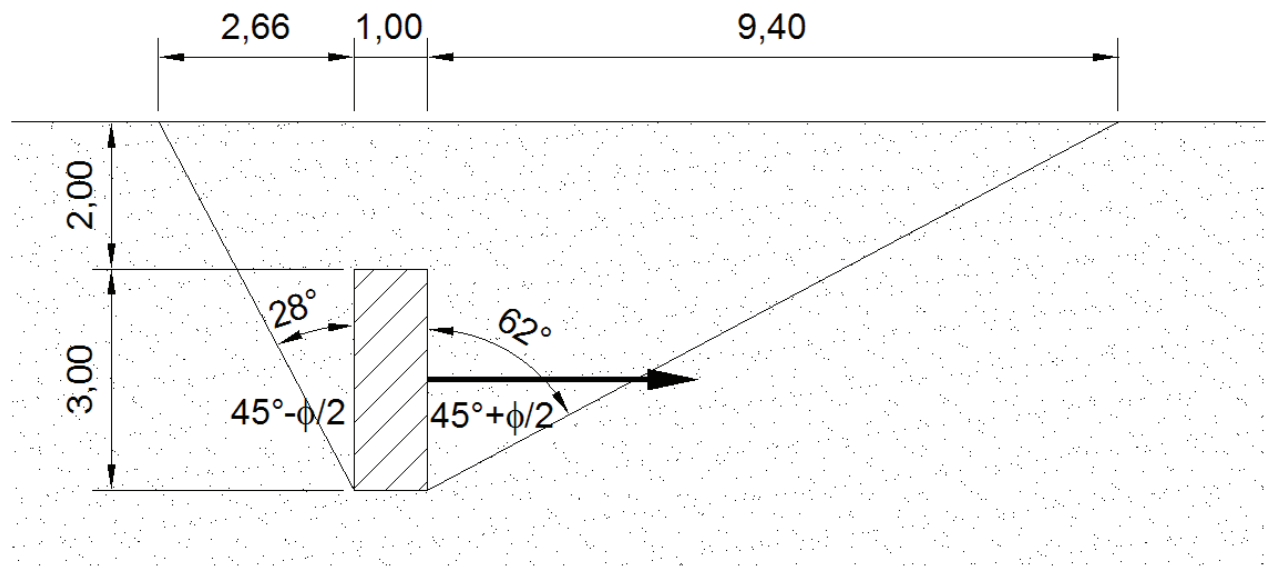

Figure 5: Range of passive and active zones near the anchor block for $\mathrm{z}_{0}=2 \mathrm{~m}$.

3D isoparametric elements with the $1^{\text {st }}$ order interpolation function [5]. The block was located centrally in the analysed soil mass. Nodes of the elements along boundaries of the analysed region were supported in the usual manner to restrain horizontal translations on the vertical boundaries and both horizontal and vertical translations on the bottom boundary plane.

Several variants of the geometric model have been considered. They differed in the size of the whole model as well as the size of elements. To enable any possible failure mechanism in the sand to develop and to avoid any influence of the outer boundary, the models had to be extended beyond the reach of the passive pressure developed in front of the anchor block as the tendon pulls against it. In the case of embedment $\mathrm{z}_{\mathrm{o}}=2 \mathrm{~m}$ and for friction angle $34^{\circ}$ the range of the passive zone is $9.40 \mathrm{~m}$ (Fig. 5). For a shallower embedment, the range is correspondingly smaller.

In FE model, the mesh refinement is required in zones of high displacement gradients. It has been noticed that the size of elements discretizing zone surrounding the anchor block affects the resulting ultimate resistance. The finer the mesh, the lower the resistance of the block was. After a few trial discretizations, the calculated ultimate resistance converged to a value that did not change any more. This final discretization was chosen for further analysis.

Finally, for the cylindrical block of diameter $D=1.0 \mathrm{~m}$, height $H=3.0 \mathrm{~m}$, the following model dimensions were adopted: $L_{x}=25 \mathrm{~m}, L_{y}=15 \mathrm{~m}$ and $L_{z}=10 \mathrm{~m}$. The meshes contained up to 31,500 finite elements and possessed over 90,000 degrees of freedom.

The simulation of the process of pulling the block was done by assuming a kinematic boundary condition for one node on the surface of the block. This node represents the point of attachment of the tendon. The horizontal displacement of the node increased to a total value of $0.80 \mathrm{~m}$ in increments of $0.01 \mathrm{~cm}$. The nodal force increases after each displacement increment. It was found that the applied displacement of $0.8 \mathrm{~m}$ is sufficient for reaching the ultimate value of pulling force (the bearing capacity of the block). 


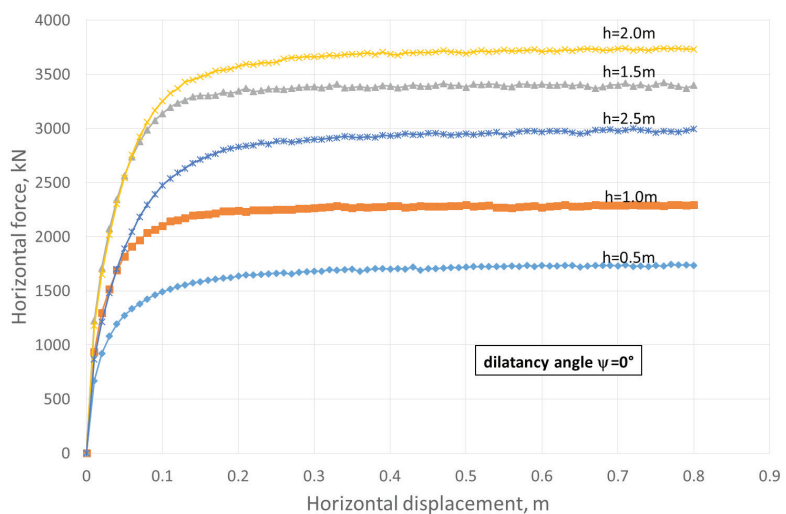

Figure 6: Force-displacement curves for the block without contact elements, surcharge thickness $\mathrm{z}_{\mathrm{o}}=2 \mathrm{~m}$, incompressible behaviour of sand at yielding and various pulling force locations.

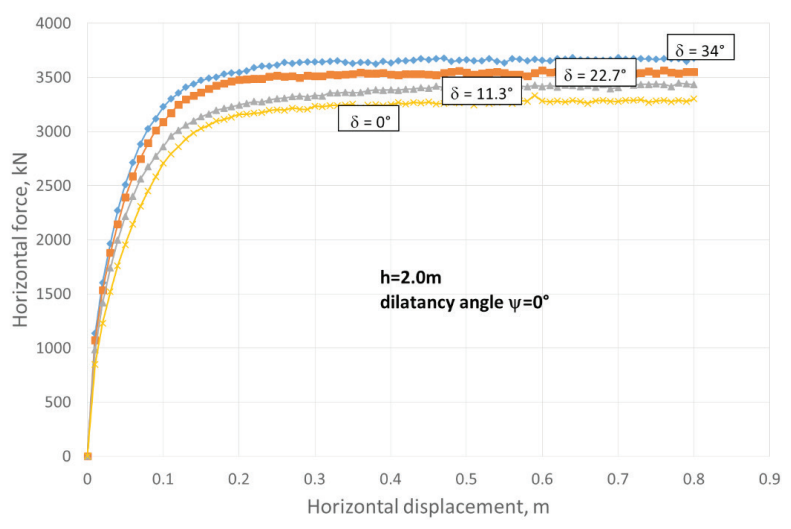

Figure 7: Force-displacement curves for the model with contact elements of different friction angle $\delta$, surcharge thickness $z_{0}=2 \mathrm{~m}$, incompressible behaviour of sand at yielding and the pulling force location $\mathrm{h}=2 \mathrm{~m}$.

\section{Finite element analysis}

In the analysis, both full contact of sand with the concrete block and sliding on block's surface was assumed. With the use of computer program Z_Soil, the value of the horizontal ultimate force pulling out the cylindrical concrete block was calculated. The ultimate force was determined based on the diagram of the 'forcedisplacement' relationship. For elastic - perfectly plastic Mohr-Coulomb model the 'force-displacement' curve increases monotonically to the ultimate value. In Fig. 6, there are five curves corresponding to the increasing pullout force for the case of the block without contact elements. In Fig. 7, there are curves obtained for the model with contact elements of different friction angles $0^{\circ}, 1 / 3 \Phi, 2 / 3 \Phi$, $\Phi$ and the location of imposing horizontal displacement $2 \mathrm{~m}$ below the block's top.
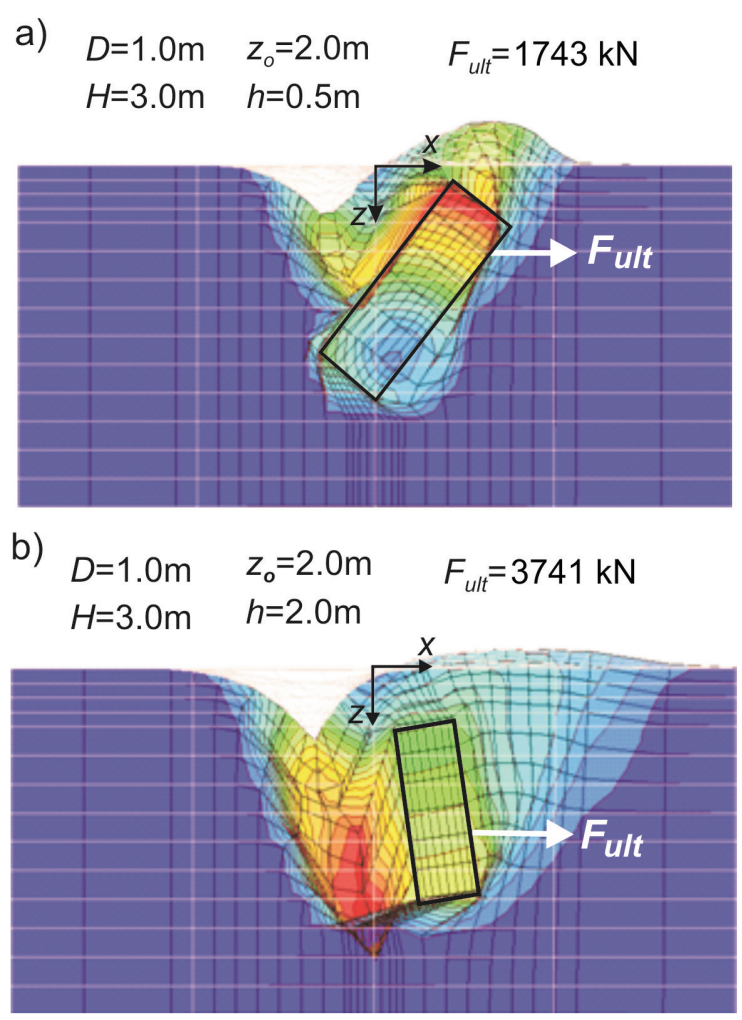

Figure 8: Block's displacement and mesh deformation in the ultimate state: a) $h=0.5 \mathrm{~m}$; b) $h=2.0 \mathrm{~m}$.

The computations were carried out for $z_{o}=1$ and $2 \mathrm{~m}$, while $h=0.5 ; 1.0 ; 1.5 ; 2.0 ; 2.5 \mathrm{~m}$. For $h<2 \mathrm{~m}$, the cylindrical concrete block pulled out by the horizontal force displaced in the direction of the force rotated clockwise, whereas for $h \geq 2.0 \mathrm{~m}$ during the displacement it rotated anti-clockwise. It is crucial to notice that when $h=2.0 \mathrm{~m}$, the block's rotation was minimized during pull out. The block's displacement, the mesh deformation surrounding the block in the plane of symmetry $\psi=0$ and the value of the ultimate force $\left(F=F_{u l t}\right)$ are shown in Fig. 8 for $h=0.5 \mathrm{~m}$ and $2.0 \mathrm{~m}$.

The value of ultimate pullout force differs significantly depending on the place of its action. Fig. 9 shows the values of ultimate force for the thickness of overburden layer $\mathrm{z}_{\mathrm{o}}=1 \mathrm{~m}$, different contact conditions and varying dilatancy at yielding. Similar results for $z_{0}=2 \mathrm{~m}$ are presented in Fig. 10.

The block embedment significantly affects the value of the ultimate force. For the overburden thickness $z_{o}=1.0 \mathrm{~m}$, the pullout force is approximately $60 \%$ of the force calculated for embedment $z_{o}=2.0 \mathrm{~m}$.

The ultimate pullout force increases with the increase of the dilatancy angle $\psi$. This trend explains the increase of the block's resistance with the compaction of sand 

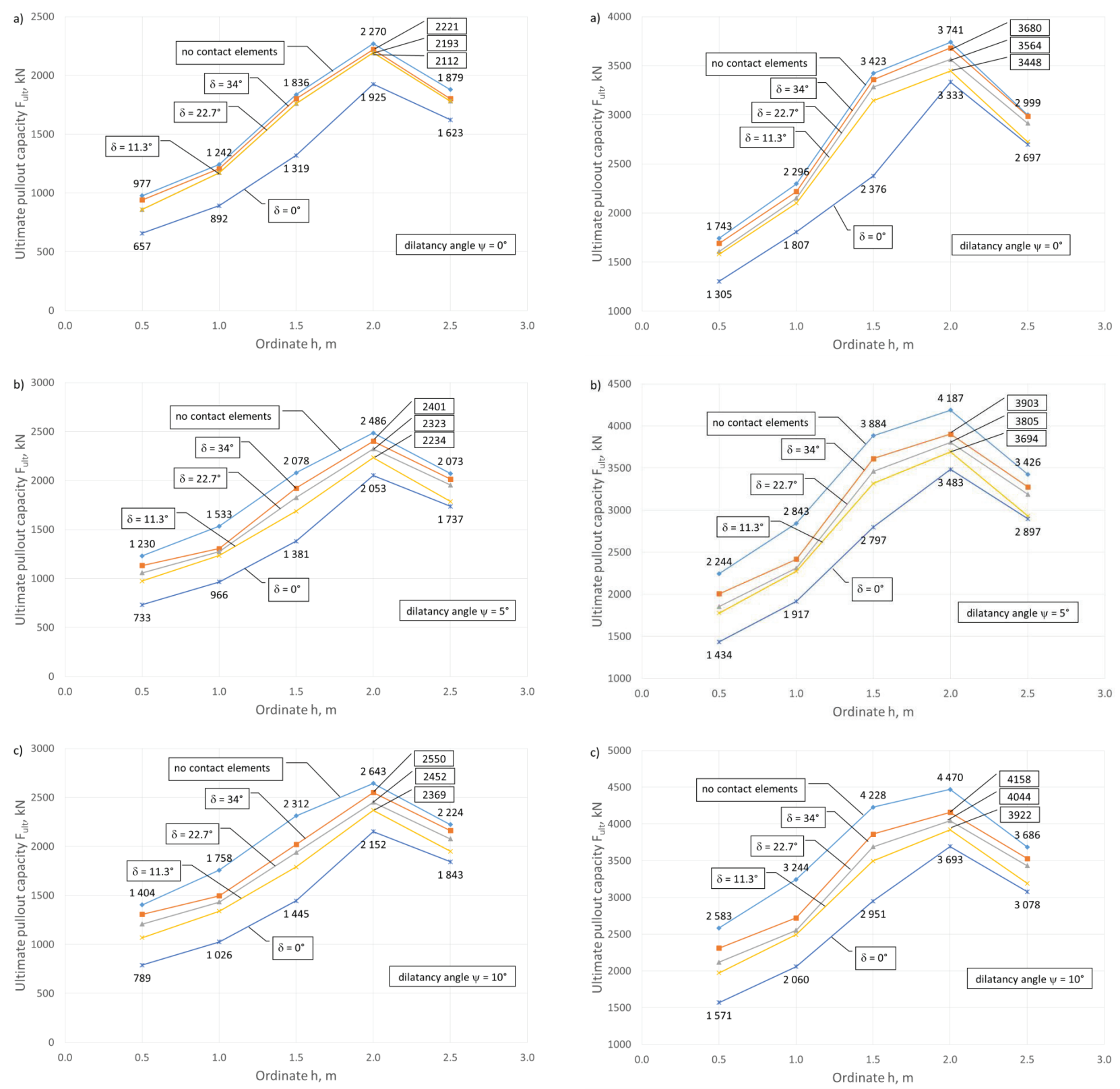

Figure 9: Variations of the ultimate force value with respect to its location and contact conditions for $\mathrm{z}_{\mathrm{o}}=1 \mathrm{~m}$.

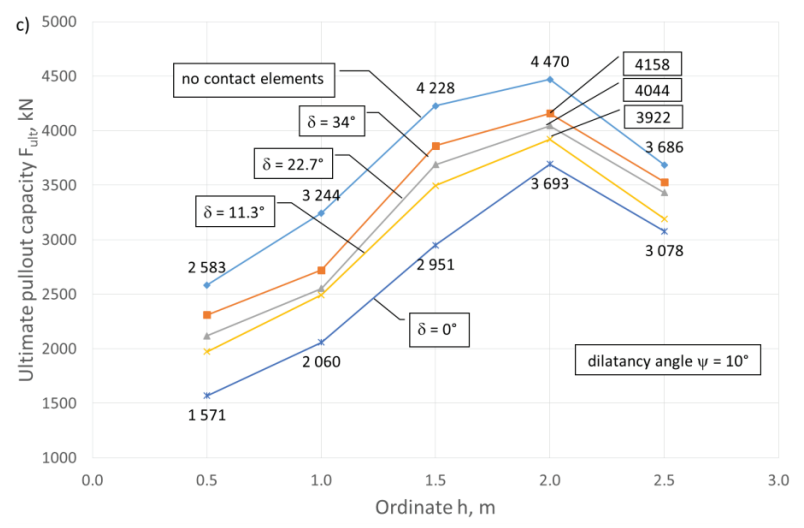

Fig. 10. Variations of the ultimate force value with respect to its location and contact conditions for $z_{0}=2 \mathrm{~m}$.

surrounding the block. The higher the compaction, the higher is $\psi$ for the same critical value of friction angle [17-20].

In each considered case, the maximum ultimate value of the horizontal force pulling out the block was achieved for $h=2.0 \mathrm{~m}$, which is $2 / 3$ of block's height. This is the location of the pulling force for which rotation is minimized and the block mainly translates in the direction of the acting force. This finding confirms the recommendation given in [21] that the tendon or tie-rod

connection to the anchor block should be located at the place where the resultant earth pressure is acting. The clockwise rotation of the block $(h<2.0 \mathrm{~m})$ significantly reduces the pullout force. As can be seen from Figs 9 and 10 , extreme values of the ultimate force were calculated for the full contact (no interface elements) and contact friction angle $\delta=0^{\circ}$ and for the force location $\mathrm{h}=0.5 \mathrm{~m}$ (the minimum ultimate force) and $2.0 \mathrm{~m}$ (the maximum ultimate force). Hence, when designing cylindrical block anchors, it is very important to eliminate the block's 
rotation. Percentage reduction for the extreme values are given in Tables 2 and 3.

The highest values of the ultimate force for all $h$ were obtained for the block without contact elements. When sliding and separation is possible by the application of contact elements, the value of ultimate force decrease. The lower the friction angle for contact elements, the lower is the resistance of the block. For a higher dilatancy angle, the drop of ultimate force from the value for block without contact elements to the value for perfectly smooth block $\left(\delta=0^{\circ}\right)$ is higher. Nevertheless, when the values of ultimate force are compared for the same contact friction, higher force accompanies higher dilatancy angle.

\section{Analytical formulation}

The scheme of horizontal components of the soil pressures acting on the cylindrical block moving in the $x$ direction is shown in Fig. 11.

The horizontal components of the soil pressures may be calculated from the formulae:

$$
\begin{aligned}
& e_{a h}=\mu_{a} \gamma z K_{a n}, \\
& e_{p h}=\mu_{p} \gamma z K_{p n},
\end{aligned}
$$

where $m_{a}$ and $m_{p}$ are coefficients of influence of the cylindrical block shape and its embedment obtained from model tests and statistical calculations [2], whereas $K_{a n}$ and $K_{p n}$ are coefficients of the normal active and passive pressures on the retaining wall respectively [16]. For the vertical block, these are the horizontal components of soil pressures. The values of coefficients $m_{a}$ and $m_{p}$ are given in Table 4 [2].

For the vertical wall, horizontal ground surface and the angle of friction between the wall and the soil $\delta$

$$
K_{a n}=\frac{1 \pm \sin \Phi \sin \left(2 m_{w} \pm \Phi\right)}{1 \mp \sin \Phi \sin \left(2 m_{t} \pm \Phi\right)} \exp ( \pm 2 v \tan \Phi),
$$

where the angles $m_{t}, m_{w}$ and $v$ are calculated from equations:

$$
\begin{gathered}
2 m_{t}=\frac{\pi}{2} \mp \Phi, \\
2 m_{w}=\cos ^{-1}\left(\frac{\sin \delta}{\sin \Phi}\right) \mp \Phi \mp \delta,
\end{gathered}
$$

Table 2: Influence of tendon attachment location and dilatancy on

\begin{tabular}{|c|c|c|c|c|}
\hline$\psi$ & $\begin{array}{l}\text { contact } \\
\text { conditions }\end{array}$ & $\begin{array}{l}F_{\text {ult } h=0.5 \mathrm{~m}}, \\
\mathrm{kN}\end{array}$ & $\begin{array}{l}F_{\text {ulth } h=2 m}, \\
k N\end{array}$ & $\mathrm{~F}_{\text {ulth }=0.5} / \mathrm{F}_{\text {ult h }=2} \times 100, \%$ \\
\hline \multirow[t]{2}{*}{$0^{\circ}$} & $\begin{array}{l}\text { no contact } \\
\text { elements }\end{array}$ & 1743 & 3741 & $46.6 \%$ \\
\hline & $\delta=0^{\circ}$ & 1305 & 3333 & $39.2 \%$ \\
\hline \multirow[t]{2}{*}{$5^{\circ}$} & $\begin{array}{l}\text { no contact } \\
\text { elements }\end{array}$ & 2244 & 4187 & $53.6 \%$ \\
\hline & $\delta=0^{\circ}$ & 1434 & 3483 & $41.2 \%$ \\
\hline \multirow[t]{2}{*}{$10^{\circ}$} & $\begin{array}{l}\text { no contact } \\
\text { elements }\end{array}$ & 2583 & 4470 & $57.8 \%$ \\
\hline & $\delta=0^{\circ}$ & 1571 & 3693 & $42.5 \%$ \\
\hline
\end{tabular}
ultimate force for $z_{0}=1 \mathrm{~m}$.

\begin{tabular}{lllll}
\hline$\psi$ & $\begin{array}{l}\text { contact } \\
\text { conditions }\end{array}$ & $\begin{array}{l}\mathbf{F}_{\text {ult h }=0.5}, \\
\mathbf{k N}\end{array}$ & $\begin{array}{l}\mathbf{F}_{\text {ult h }=2 \mathrm{~m}}, \\
\mathbf{k N}\end{array}$ & $\mathbf{F}_{\text {ult h }=0.5} / \mathbf{F}_{\text {ult h }=2} \times 100, \%$ \\
\hline $0^{\circ}$ & $\begin{array}{l}\text { no contact } \\
\text { elements }\end{array}$ & 977 & 2270 & $43.0 \%$ \\
& $\begin{array}{l}\delta=0^{\circ} \\
5^{\circ}\end{array}$ & 657 & 1925 & $34.1 \%$ \\
& $\begin{array}{l}\text { no contact } \\
\text { elements } \\
\delta=0^{\circ}\end{array}$ & 1230 & 2486 & $49.5 \%$ \\
$10^{\circ}$ & 733 & 2053 & $35.7 \%$ \\
& $\begin{array}{l}\text { no contact } \\
\text { elements } \\
\delta=0^{\circ}\end{array}$ & 1404 & 2643 & $53.1 \%$ \\
\hline
\end{tabular}

Table 3: Influence of tendon attachment location and dilatancy on ultimate force for $z_{0}=2 \mathrm{~m}$.

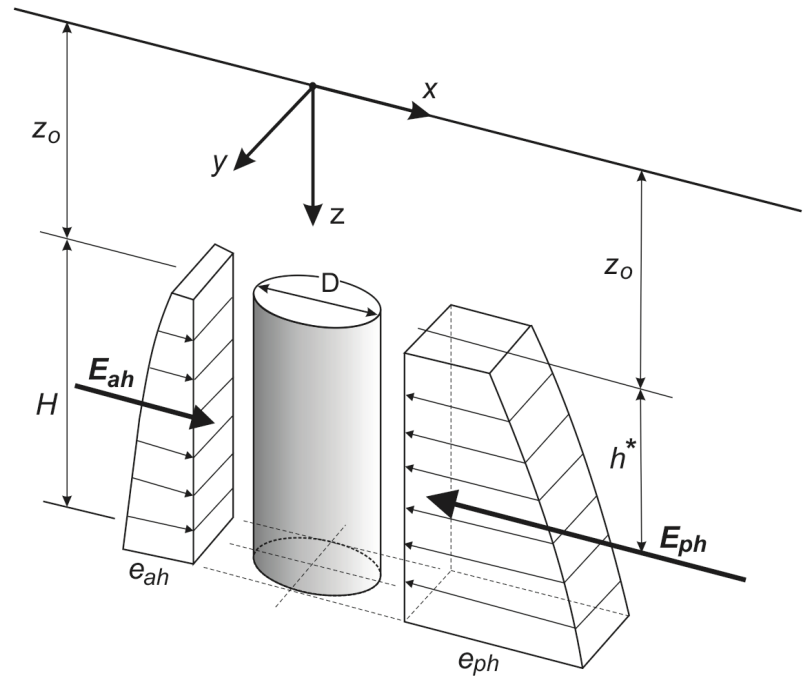

Figure 11: Scheme of pressures on the block. 
Table 4: Coefficients $m_{a}$ and $m_{p}$.

\begin{tabular}{lllllll}
\hline$z / D$ & $\mathbf{0}$ & $\mathbf{2}$ & $\mathbf{4}$ & $\mathbf{6}$ & $\mathbf{8}$ & $\mathbf{1 0}$ \\
\hline$m_{a}$ & 1 & 0.68 & 0.46 & 0.33 & 0.26 & 0.22 \\
$m_{p}$ & 1 & 1.64 & 2.32 & 2.90 & 3.56 & 4.0 \\
\hline
\end{tabular}

Table 5: Coefficients of earth pressure for different contact friction.

\begin{tabular}{lll}
\hline$\delta$ & $\mathrm{K}_{\mathrm{pn}}$ & $\mathrm{K}_{\mathrm{an}}$ \\
\hline $0^{\circ}$ & 3.5371 & 0.2827 \\
$1 / 3 \phi$ & 4.8707 & 0.2577 \\
$2 / 3 \phi$ & 6.0623 & 0.2395 \\
$\phi$ & 6.7123 & 0.2280 \\
\hline
\end{tabular}

Table 6: Passive earth pressure for different contact friction and embedment $z_{0}=1 \mathrm{~m}$.

\begin{tabular}{llll}
\hline$\delta$ & $\mathbf{e}_{\mathrm{p} 1}, \mathbf{k P a}$ & $\mathbf{e}_{\mathrm{p} 2}, \mathbf{k P a}$ & $\mathrm{E}_{\mathrm{ph}}, \mathbf{k N}$ \\
\hline $0^{\circ}$ & 84.04 & 590.84 & 1012.3 \\
$1 / 3 \Phi$ & 115.73 & 813.60 & 1394.0 \\
$2 / 3 \Phi$ & 144.04 & 1012.65 & 1735.0 \\
$\Phi$ & 159.48 & 1121.23 & 1921.1 \\
\hline
\end{tabular}

Table 7: Passive earth pressure for different contact friction and embedment $z_{0}=2 \mathrm{~m}$.

\begin{tabular}{llll}
\hline$\delta$ & $\mathbf{e}_{\mathrm{p} 1}, \mathbf{k P a}$ & $\mathbf{e}_{\mathrm{p} 2}, \mathbf{k P a}$ & $\mathrm{E}_{\mathrm{ph}}, \mathbf{k N}$ \\
\hline $0^{\circ}$ & 208.83 & 830.87 & 1559.6 \\
$1 / 3 \Phi$ & 287.56 & 1144.12 & 2147.5 \\
$2 / 3 \Phi$ & 357.92 & 2672.94 & 2672.9 \\
$\Phi$ & 396.30 & 1576.72 & 2959.5 \\
\hline
\end{tabular}

$$
v=m_{t}-m_{w}
$$

In (5) - (8) $\Phi$ and $\delta$ are given in radians. Upper signs should be used for calculating passive pressure and lower signs for calculating active pressure [16].

Values of $K_{p n}$ and $K_{a n}$ are given in Table 5. As the active pressure is much lower than passive pressure, it can be neglected in engineering estimations.

Due to the non-linear relationship between the coefficient $m_{p}$ and $z / D$, the distribution of passive pressure is also non-linear. Assuming linear distribution of pressures over the height of the block, we can make a safe estimation and easily calculate the resultant horizontal component $E_{p h}$ of passive pressure acting on the block, which is approximately equal to the ultimate value of the horizontal force pulling out the block. Value of $E_{p h}$ is obtained in a usual manner by integrating the passive pressure over the height of the block, which, in case of the linear distribution between $\mathrm{e}_{\mathrm{p} 1}$ at the top and $\mathrm{e}_{\mathrm{p} 2}$ at the bottom of the block, is the area of a trapezoid of height $H$.

Comparison of ultimate forces obtained from the finite element calculations for the most conservative case of incompressible plastic flow $(\psi=0)$ with analytical results reveals that the analytical method recommended in [22] yields significantly lower forces for all considered contact friction, which makes the analytical prediction very conservative. Detailed comparison is given in Table 8.

The resultant passive pressure is situated at the distance of $1.88 \mathrm{~m}$ for $z_{o}=1.0 \mathrm{~m}$ and $1.80 \mathrm{~m}$ for $z_{o}=2.0 \mathrm{~m}$ from the top of the block. Thus, the presented engineering approximation can be used only for blocks of small embedment [2] without vertical displacement in soil.

Table 8: Comparison of ultimate pullout forces for different contact friction and embedment $z_{0}$.

\begin{tabular}{lllllll}
\hline & $\mathbf{Z}_{\mathbf{0}}=\mathbf{1 m}$ & & $\mathbf{Z}_{\mathbf{o}}=\mathbf{2 m}$ \\
$\delta$ & $\mathbf{F}_{\text {ult anal, }} \mathbf{k N}$ & $\mathbf{F}_{\text {ult FEM, }}, \mathbf{k N}$ & $\mathbf{F}_{\text {ult anal }} / \mathbf{F}_{\text {ult FEM }} \times \mathbf{1 0 0 , \%}$ & $\mathbf{F}_{\text {ult FEM, }} \mathbf{k N}$ & $\mathbf{F}_{\text {ult anal, }}, \mathbf{k N}$ & $\mathbf{F}_{\text {ult FEM }} / \mathbf{F}_{\text {ult anal }} \times 100, \%$ \\
\hline$\Phi$ & 1921.1 & 2221 & $86 \%$ & 2959.5 & 3680 & $80 \%$ \\
$2 / 3 \Phi$ & 1750.03 & 2193 & $79 \%$ & 2672.9 & 3564 & $75 \%$ \\
$1 / 3 \Phi$ & 1394.0 & 2112 & $66 \%$ & 2147.5 & 3448 & $62 \%$ \\
$0^{\circ}$ & 1012.3 & 1925 & $53 \%$ & 1559.6 & 3333 & $47 \%$ \\
\hline
\end{tabular}




\section{Conclusions}

The ultimate value of the cylindrical pulled out block depends on its dimensions, depth of embedment and points of pullout force application. The maximum resistance of a single block is acquired when the block is displaced horizontally without rotation. Significantly smaller resistance is acquired when the block rotates during displacement. By making use of formulae accessible in the literature, we can only find solution to the cylindrical block displacement without rotation, and the pullout resistance is at least $24 \%$ smaller than the resistance calculated numerically.

Having introduced the contact elements with friction angle $\delta=\Phi$ in the finite element program, the resulting ultimate pullout force decreases with respect to the models without contact elements.

The analysis shows that when designing cylindrical concrete anchors, it is absolutely necessary to ensure block displacement without rotation. This kind of displacement is easiest to acquire by the application of two horizontal flexible tendons attached to the block.

\section{References}

[1] Briaud J.-L. \& Kim N.K. (1998). Beem Column Method for Tieback Walls. J. Geotech. Geoenviron. Eng. 124(1), 67-69. DOI: 10.1061/(ASCE)1090-0241(1998)124:1(67).

[2] Smoltczyk U. (2003). Geotechnical Engineering Handbook, Vol. 1. Fundamentals. Ernest \& Sohn, Berlin.

[3] Smith I. (2006). Smith's Elements of Soil Mechanics. Blackwell Publishing Ltd, Oxford, UK.

[4] Briaud J.-L. (2013). Geotechnical Engineering Unsaturated and Saturated Soils. John Wiley \& Sons Inc., Hobokan, New Jersey.

[5] Zimmermann Th., Truty A., Urbański A. \& Podleś K. (2010). Z_Soil. PC 2010 3D user manual, Theory, Tutorials and Benchmarks, Data Preparation. Elmepress Int. \& Zace Services Ltd, Switzerland.

[6] Rowe R.K. \& Davis E.H. (1982). The behaviour of anchor plates in sand. Géotechnique. 32(1). 25-41. DOI: 10.1680/ geot.1982.32.1.25.

[7] Murray E.I. \& Geddes I.D. (1989). Resistance of passive inclined anchors in cohesionless medium. Géotechnique. 39(3). 417431.
[8] Kumar I. (2002). Seismic horizontal pullout capacity of vertical anchors in sands. Canadian Geotechnical Journal. 39(4). 982991. DOI: 10.1139/t02-021.

[9] Merifield R.S. \& Sloan S.W. (2006). The ultimate pullout capacity of anchors in frictional soils. Canadian Geotechnical Journal. 43(8). 852-868. DOI: 10.1139/t06-052.

[10] Kame G.S., Dewaikar D.M. \& Deepankar Ch. (2012). Pullout Capacity of a Vertical Plate Anchor Embedded in Cohesion-less Soil. Earth Science Research. 1(1). 27-56. DOI: 10.5539/esr. v1n1p27.

[11] Bhattacharya P. \& Kumar I. (2014). Pullout capacity of inclined plate anchors embedded in sand. Canadian Geotechnical Journal. 51(6). 1365-1370. DOI: 10.1139/cgj-2014-0114.

[12] Bhattacharya P. (2016). Pullout capacity of strip plate anchor in cohesive sloping ground under undrained condition, Comp. Geotech. 78. 134-143. DOI: 10.1016/j.compgeo.2016.05.006.

[13] Nouri H., Biscontin G. \& Aubeny C.P. (2017). Numerical prediction of undrained response of plate anchors under combined translation and torsion, Comp. Geotech. 81. 39-48. DOI: 10.1016/j.compgeo.2016.07.008.

[14] Desai Ch.S. \& Zaman M. (2014). Advanced Geotechnical Engineering. Soil - Structure Interaction Using Computer and Material Models. Taylor \& Francis Group, Boca Raton, FL, USA.

[15] Stutz H., Wuttke F. \& Benz T. (2014). Extended zero-thickness interface element for accurate soil - pile interaction modelling, In: Numerical Methods in Geotechnical Engineering, Hicks, Brinkgreve \& Royce (Eds). Taylor \& Francis Group, London, 283-288.

[16] Bond A. \& Harris A. (2010). Decoding Eurocode. Taylor and Francis, New York, USA.

[17] Schofield A. N. \& Wroth C. P. (1968). Critical State Soil Mechanics. McGraw-Hill.

[18] Atkinson J.H. (2007). The mechanics of soils and foundations. Taylor \& Francis, London and New York.

[19] Flora, A. \& Modoni G. (1997). Upgrading Equipment and Procedures for Stress Path Triaxial Testing of Coarse-Grained Materials. Geotechnical Testing Journal. Vol. 20., No. 4. 459469. DOI: 10.1520/GTJ10412J.

[20] Iolli S., Modoni G., Chiaro G. \& Salvatore E. (2015). Predictive correlations for the compaction of clean sands. Transportations Geotechnics. 4. 38-49. DOI: 10.1016/j.trgeo.2015.06.004.

[21] Geotechnnical Engineering Handbook (2011). Edited by Braja M. Das, J. Ross Publishing, Inc., Fort Lauderdale.

[22] EN 1997-1. (2004). Eurocode 7: Geotechnical design - Part 1. General rules. Brussels. 